\title{
THE ANGLE OF AN OPERATOR AND POSITIVE OPERATOR PRODUCTS ${ }^{1}$
}

\author{
BY KARL GUSTAFSON
}

\author{
Communicated by F. E. Browder, December 5, 1967
}

1. Introduction. Let $A \geqq 0$ and $B \geqq 0$ be two positive bounded selfadjoint operators. The two algebraic questions which immediately arise are: (1) is $A+B \geqq 0 ;(2)$ is $B A \geqq 0$ ? The first question (and its extension to accretive operators on a Banach space) has trivially an affirmative answer; the second question also has an affirmative answer, under the additional condition that $A$ and $B$ commute. However, apparently question (2), which due to the general nonselfadjointness of $B A$ must be reformulated as $\left(2^{\prime}\right) \operatorname{Re}(B A x, x) \geqq 0$ for all $x$, has remained unanswered for general (i.e., noncommuting) operators $A$ and $B$. This is clearly an important mathematical question, and the purpose of this announcement is to state sufficient (and rather sharp) conditions for the more general $\left(2^{\prime \prime}\right) \operatorname{Re}[B A x, x] \geqq 0$ for all $x$, where $[x, y]$ is a semi-inner product on any Banach space (in the special case of a Hilbert space, it is necessarily the inner product).

In $\$ 2$ we introduce two new quantities which are technically essential in our treatment, namely the angle of an operator and the minimum of a certain (norm) function related to tangent functionals. In $\$ 3$ we bound the behavior of the latter, determining exact values for the important class of selfadjoint operators. Using these two quantities, in $\$ 4$ we give criteria for $\left(2^{\prime \prime}\right)$ to hold; our main result is Theorem 4.2. In $\$ 5$ we give positive lower bounds for the resulting accretive operator products, and compare them with bounds for commuting selfadjoint operators. In $\$ 6$ we apply these results to a semigroup question which motivated this work. However, it is expected that the criteria here developed will be useful elsewhere in operator theory and in those parts of theoretical physics where a zero commutator is the exception. In $\$ 7$ we make further comments. Complete proofs of these and related results may be found in the references (in particular, [6]) and in a paper under preparation.

2. Definitions, the angle of an operator. Consider the following (well-known) quantitative functions defined on the Banach algebra of bounded operators $B: X \rightarrow X, X$ a complex Banach space, $[x, y]$ a semi-inner product (see Lumer [11]), $\|x\|=1:\|B\|=\sup \|B x\|$;

${ }^{1}$ Partially supported by NSF GP $7041 \mathrm{X}$, and by Battelle Institute, Geneva. 
$\theta(B)=\sup \operatorname{Re}[B x, x] ; \gamma(B)=\inf \|B x\| ; m(B)=\inf \operatorname{Re}[B x, x]$. An operator $B$ is called accretive if $m(B) \geqq 0$, strongly accretive if $m(B)>0$, dissipative if $\theta(B) \leqq 0$, strongly dissipative if $\theta(B)<0$. If $B$ is selfadjoint ( $X$ a Hilbert space), replace accretive and dissipative by positive and negative, respectively, and note that $M(B)=\theta(B)$ is commonly used then. We will also consider unbounded operators, the above quantities remaining meaningful in the extended real numbers. Hereafter an operator $B$ is assumed bounded with $D(B)=X$, unless specifically stated otherwise; in all cases, $D(B)$ is assumed to be dense in $X$.

Definition 1. $\phi_{R}(B)=\operatorname{Cos}^{-1}\left\{\inf _{x}\left(\operatorname{Re}[B x, x] \cdot\|B x\| \|^{-1}\right), x \in D(B)\right.$, $\|x\|=1, x \in N(B)\}$. Here $B$ may be bounded or unbounded. We call $\phi_{R}(B)$ the (real) angle of $B$; it measures the maximum (real) turning effect of $B$.

Definition 2. $g_{m}(B)=\min _{\epsilon} g(\epsilon, B), \epsilon \geqq 0, g(\epsilon, B)=\|\epsilon B+\mathrm{I}\|$. Let $\epsilon_{m}$ (unique when $X$ is a Hilbert space and $\theta(B)<0$ ) denote any value of $\boldsymbol{\epsilon}$ such that $g\left(\epsilon_{m}, B\right)=g_{m}(B)$. Clearly $g_{m}(B)=1$ when $\theta(B) \geqq 0$; but interest attaches to $g_{m}(B)$ when $\theta(B)<0$.

3. Lemmas. The following is a minor sharpening of results in [11], [12], [13]; for bounded everywhere defined operators on a Hilbert space, it is contained in the results of [1].

LEMma 3.1. Let $B$ (bounded or unbounded) be dissipative. Then $\gamma(\epsilon B-I) \geqq 1-\epsilon \theta(B), \epsilon \geqq 0$.

The next result establishes functions bounding the behavior of $g(\epsilon, B)$.

LEMMA 3.2.

(i) $\max \{\epsilon\|B\|-1,1+\epsilon \theta(B)\} \leqq g(\epsilon, B)$

$$
\leqq \min \left\{1+\epsilon\|B\|, 1+\epsilon \theta(B)+\epsilon^{2}[\|B\|+|\theta(B)|]^{2}\right\},
$$

$\theta(B) \leqq g^{\prime}(\epsilon, B) \leqq\|B\|$ for all, except at most a countable number of values of, $\epsilon$.

(ii) When $B$ is dissipative,

$$
(\|B\|+\theta(B)) \cdot(\|B\|-\theta(B))^{-1} \leqq g(\epsilon, B) \leqq\left(1+\epsilon^{2}\|B\|^{2}\right) \cdot(1-\epsilon \theta(B))^{-1} .
$$

In a Hilbert space the upper bounds in Lemma 3.2 can be sharpened, since then it is easy to see that $g(\epsilon, B) \leqq\left(\epsilon^{2}\|B\|^{2}+1+2 \epsilon \theta(B)\right)^{1 / 2}$, and this upper bound is attained by examples. A partial upper bound for $g(\epsilon, B)$ was found in Hildebrand [9] for a Hilbert space. The lower bounds in Lemma 3.2 are sharp, as seen by: 
Lemma 3.3. Let $B$ be selfadjoint. Then $g(\epsilon, B)$ assumes (everywhere) the lower bounding values of Lemma 3.2 ; if $g^{\prime}(\epsilon, B)$ has a discontinuity, it occurs at $\epsilon_{c}=2(\|B\|-\theta(B))^{-1}$; when $\theta(B)<0, \epsilon_{m}=\epsilon_{o}$ and $g_{m}(B)$ $=(\|B\|+\theta(B)) \cdot(\|B\|-\theta(B))^{-1}$.

4. Positive operator products. When the operators commute, it is easy to obtain results such as the following, which is not intended to be most general.

Proposition 4.1. Let $A$ (bounded or unbounded) be selfadjoint and positive, $B$ accretive, $A$ commuting with $B$; then $B A$ is accretive.

Our main concern is the general case of noncommuting operators in a Banach space, and our main result is the following.

Theorem 4.2. Let $A$ (bounded or unbounded) and $B$ be such that $g_{m}(-B) \leqq \cos \phi_{R}(A)$; then $B A$ is accretive.

Corollary 4.3. Let $A$ and $B$ be strongly accretive. If either $g_{m}(-B)$ $\leqq m(A) \cdot\|A\|^{-1}$ or $g_{m}(-A) \leqq m(B) \cdot\|B\|^{-1}$, then both $B A$ and $A B$ are accretive.

Corollary 4.4. Let $A$ and $B$ be positive selfadjoint operators. If $(M(B)-m(B)) \cdot(M(B)+m(B))^{-1} \leqq m(A) \cdot(M(A))^{-1}$, or (equivalently) vice-versa, then both $B A$ and $A B$ are accretive. Sharper: $B A$ is accretive if l.h.s. $\leqq 2(m(A) \cdot M(A))^{1 / 2} \cdot(m(A)+M(A))^{-1}$.

It can be seen by one-dimensional normal operators and twodimensional selfadjoint operators that the above criteria is sharp. It should be noted that $A$ strongly positive and $B$ strongly positive does not imply $B A$ accretive, in contrast to both the commuting case (Proposition 4.1) and the results for the spectrum $\sigma(B A)$ obtained in Williams [15].

5. Lower bounds, an example. The lower bound $b_{1}$ below is obtained as the uniform lower bound of an inequality of recent interest due to Diaz and Metcalf [2]; similar inequalities have been given by Greub and Rheinboldt, Petryshyn, Makai, Krasnosel'skir and Krein, Kantorovich, etc.; see [2].

THEOREM 5.1. The following are lower bounds for $m(B A)$.

$b_{1}=\left[m(A) \cdot(m(B))^{2} \cdot\|A\|+m(B) \cdot(m(A))^{2} \cdot\|B\|\right] \cdot[m(A) m(B)+\|A\|$ $\cdot\|B\|]^{-1} ; A$ and $B$ strongly positive commuting selfadjoint operators.

$b_{2}=m(A) \cdot m(B) ; A$ (bounded or unbounded) positive selfadjoint, $B$ accretive, $A$ commuting with $B$. 
$b_{3}=m(A) \cdot\left[1-g_{m}(-B) \cdot\left(\cos \phi_{R}(A)\right)^{-1}\right] \cdot\left(\epsilon_{m}(-B)\right)^{-1} ; A$ (bounded or unbounded) and $B$ such that $g_{m}(-B) \leqq \cos \phi_{R}(A)$.

As a more accessible lower bound, $b_{3}$ has itself the lower bound $b_{4}=\left(m(A)-g_{m}(-B) \cdot\|A\|\right) \cdot\left(\epsilon_{m}(-B)\right)^{-1}$ when $A$ is bounded. For a comparison, let $A$ and $B$ be strongly positive commuting selfadjoint operators; then one can see that always $b_{4} \leqq b_{1} \leqq b_{3} \leqq b_{2}$. As an example, let $\|A\|=\|B\|=1, m(A)=m(B)=2^{-1}$; then $b_{2}=4^{-1}, b_{1}=5^{-1}$, $b_{4}=8^{-1}$. More specifically $\cos \phi_{R}(A) \cong 0.9428$ and $b_{3} \cong 0.2424$, a result practically as good as the best bound given under the commuting hypotheses.

6. Multiplication of semigroup generators. Let $G(1,0)$ denote all infinitesimal generators of strongly continuous contraction semigroups on a Banach space $X$. Much is known about the perturbation of $A \in G(1,0)$; for the most general condition on $B$ (bounded or unbounded) so that $A+B \in G(1,0)$, see [4]. That then the semigroup generated by $A+B$ may be formed as a limit of products of the semigroups generated by $A$ and $B$ has been investigated by Trotter, Nelson, Kato; see [10], [13]. However, the question of when the product $B A \in G(1,0)$ was apparently first considered in the recent paper Dorroh [3], where it was shown that if $X$ is a space of bounded functions and $A \in G(1,0)$, then $p A \in G(1,0)$ when $p$ is the operator given by multiplication by a positive bounded function, bounded below away from zero. In [6] the following is established.

Theorem 6.1. Let $X$ be any Banach space, $A$ (bounded or unbounded) $\in G(1,0)$, either of the following (disjoint) conditions holding for $B$ :

(i) $B$ is strongly accretive;

(ii) $\exists \epsilon>0$ such that $\epsilon\left\|B^{-1}\right\|-1>\|\epsilon B-I\| \geqq 1, m(B) \leqq 0$. Then $B A \in G(1,0)$ if and only if $B A$ is dissipative.

Corollary 6.2. Let $A$ (bounded or unbounded) $\in G(1,0), B$ strongly accretive, $g_{m}(-B) \leqq \cos \phi_{R}(-A)$; then $B A \in G(1,0)$.

7. Further remarks. Other trigonometric interpretations are possible; e.g., for positive selfadjoint $A$ with point spectrum only, one has $g_{m}(-A)=\sin \phi_{R}(A)$. The results herein are somewhat constrained to bounded $A$ by the interesting fact [8] that of ten (e.g., for most Hilbert space operators) accretive unbounded $A$ possess the property $\cos \phi_{R}(A)=0$. The two parameters $\cos \phi_{R}(A)$ and $g_{m}(B)$ are currently being computed for certain operator classes. Extensions of the results of $\S 6$ to unbounded $B$, and applications thereof, will appear in [7]. W. G. Strang has kindly observed that our criteria contains his previous results [14] for $A B+B A \geqq 0, A$ and $B$ selfadjoint matrices. 


\section{REFERENCES}

1. S. K. Berberian and G. H. Orland, On the closure of the numerical range of an operator, Proc. Amer. Math. Soc. 18 (1967), 499-503.

2. J. B. Diaz and F. T. Metcalf, Complementary inequalities. III: Inequalities complementary to Schwarz's inequality in Hilbert space, Math. Ann. 162 (1965), 120139.

3. J. R. Dorroh, Contraction semigroups in a function space, Pacific J. Math. 19 (1966), 35-38. 338.

4. K. E. Gustafson, $A$ perturbation lemma, Bull. Amer. Math. Soc. 72 (1966), 334-

5. - $A$ note on left multiplication of semigroup generators, Pacific J. Math. (to appear).

6. - Positive (noncommuting) operator products and semigroups, Math. Zeitschrift (to appear).

7. K. E. Gustafson and G. Lumer, Multiplicative perturbation of semigroups, (in preparation).

8. K. E. Gustafson and B. Zwahlen, On the cosine of unbounded operators, (in preparation).

9. S. Hildebrandt, Ueber den numerischen Wertebereich eines Operators, Math. Ann. 163 (1966), 230-247. 1966.

10. T. Kato, Perturbation theory for linear operators, Springer-Verlag, Berlin,

11. G. Lumer, Semi inner-product spaces, Trans. Amer. Math. Soc. 100 (1961), 29-43.

12. G. Lumer and R. S. Phillips, Dissipative operators in a Banach space, Pacific J. Math. 11 (1961), 679-698.

13. E. Nelson, Feynman integrals and the Schrödinger equation, J. Math. Phys. 5 (1964), 332-343.

14. W. Gilbert Strang, Eigenvalues of Jordan products, Amer. Math. Monthly 69 (1962), 37-40.

15. J. P. Williams, Spectra of products and numerical ranges, J. Math. Anal. Appl. 17 (1967), 214-220.

University OF MinNesota 